\title{
Boundary element modeling of curved wire antenna configurations
}

\author{
D. Poljak, V. Doric \& F. Lerinc \\ University of Split, Croatia
}

\begin{abstract}
The paper deals with an analysis of curved wire antennas. The formulation is based on the Pocklington integro-differential equations for curved wires which is solved via the Galerkin-Bubnov scheme of the Indirect Boundary Element method (GB-IBEM). Some illustrative computational examples related to thin wire loop antenna and various helical antenna types are given in the paper.

Keywords: boundary elements, curved wires, integral equations, loop antennas, helical antennas.
\end{abstract}

\section{Introduction}

Curved wire antennas, such as loops or helical antennas, have a number of applications in communication systems. Thus, antennas used with portable transceivers at very high frequencies are usually in the form of helix, mounted on a radio case. Of great interests are various configurations of helical antennas either as individual elements, or as parts of antenna arrays. In particular, helical antennas operate in various modes and two principal modes are the normal (broadside) and axial (endfire) modes [1,2].

The normal mode radiation occurs when the helical antenna diameter is much smaller than wavelength, while the axial mode occurs when the helix circumference is one wavelength and ensures maximum radiation along the helical antenna axis. The helix axial mode is often of most importance and it is used in wide range of applications [3].

The present work deals with boundary element modeling of certain curved wire configurations. The formulation is based on the Pocklington integrodifferential equation for curved wires [4]. 
The corresponding Pocklington equation is solved via the Galerkin-Bubnov variant of the Boundary Element method (GB-IBEM) [5].

Some illustrative computational examples pertaining to thin wire loop antenna and some helix configurations are given in the paper.

\section{Formulation}

The geometry of interest is generally related to a curved wire configuration in unbounded lossless medium, as shown in Figure 1.

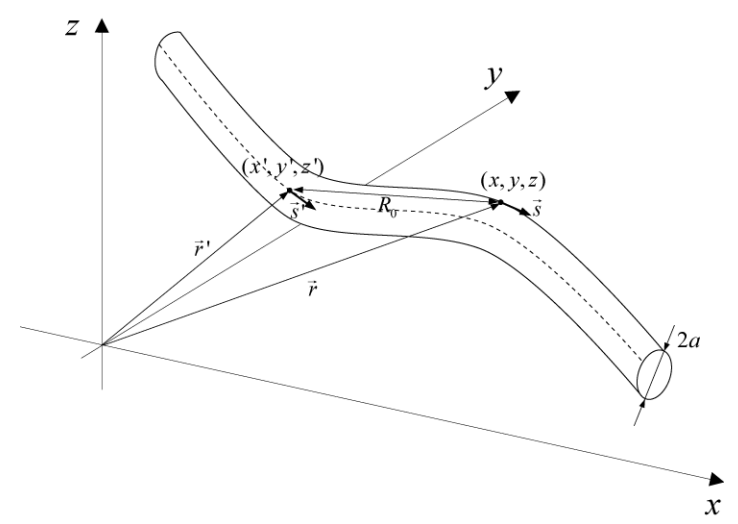

Figure 1: Wire configuration of arbitrary shape.

The corresponding Pocklington integro-differential equation for curved wires can be derived from Maxwell equations by featuring the continuity condition for the tangential components of the electric field at perfectly conducting (PEC) wire surface [4] and is given by

$$
E^{i n c}(s)=-\frac{1}{j 4 \pi \omega \varepsilon_{0}} \int_{0}^{L}\left[k_{1}^{2} \vec{e}_{s^{2}} \vec{s}_{s^{\prime}}-\frac{\partial^{2}}{\partial s \partial s^{\prime}}\right] g_{0}\left(s, s^{\prime}\right) I\left(s^{\prime}\right) d s^{\prime}
$$

where $I\left(s^{\prime}\right)$ is the unknown current distribution along the wire, $\vec{E}^{\text {inc }}$ stands for the incident field, $\vec{e}_{s}$ and $\vec{e}_{s^{\prime}}$ is the unit vector tangential to the wire surface and axis respectively, while $g_{0}\left(s, s^{\prime}\right)$ denotes the Green function

$$
g_{0}\left(s, s^{\prime}\right)=\frac{e^{-j k R}}{R}
$$

and $R$ is the distance from the source point to the observation point, respectively. The propagation constant of free space is:

$$
k=\omega \sqrt{\mu_{0} \varepsilon_{0}}
$$

Once the current distribution is determined the electric field radiated by curved wire structure can be determined from expression [4]: 


$$
\begin{gathered}
\vec{E}=\frac{1}{j 4 \pi \omega \varepsilon_{0}}\left[k^{2} \int_{0}^{L} \vec{e}_{s^{\prime}} I\left(s^{\prime}\right) g_{0}\left(\vec{r}, \vec{r}^{\prime}\right) d s^{\prime}+\int_{0}^{L} \frac{\partial I\left(s^{\prime}\right)}{\partial s^{\prime}} \nabla g_{0}\left(\vec{r}, \vec{r}^{\prime}\right) d s^{\prime}\right] \\
\vec{H}_{S}=-\frac{1}{4 \pi} \int_{0}^{L} I\left(s^{\prime}\right) \vec{e}_{s^{\prime}} \times \nabla g_{0}\left(\vec{r}, \vec{r}^{\prime}\right) d s^{\prime}
\end{gathered}
$$

\section{Numerical solution}

The Pocklington integro-differential equation for curved wires (1) is handled via the GB-IBEM. Performing certain mathematical manipulation Pocklington equation (1) is transformed into the following matrix equation [4]

$$
\sum_{m=1}^{M} \sum_{\mathrm{i}=1}^{n_{e}}[Z]_{\mathrm{ji}}^{m}\{I\}_{\mathrm{i}}^{m}=\{\mathrm{V}\}_{\mathrm{j}}^{m}, \quad j=1,2, \ldots n_{e}
$$

where the mutual impedance matrix is [4]

$$
[Z]_{i j}^{m}=-\int_{-1}^{1} \int_{-1}^{1}\{D\}_{j}\left\{D^{\prime}\right\}_{i}^{T} g_{0}\left(s, s^{\prime}\right) \frac{d s^{\prime}}{d \xi^{\prime}} d \xi^{\prime} \frac{d s}{d \xi} d \xi+k^{2} \vec{e}_{s} \vec{e}_{s} \int_{-1}^{1} \int_{-1}^{1}\{f\}_{j}\left\{f^{\prime}\right\}_{i}^{T} g_{0}\left(s, s^{\prime}\right) \frac{d s^{\prime}}{d \xi^{\prime}} d \xi^{\prime} \frac{d s}{d \xi} d \xi
$$

while the voltage vector is given by [4]

$$
\{V\}_{j}^{m}=-j 4 \pi \omega \varepsilon_{0} \int_{-1}^{1} E_{s}^{i n c}(s) f_{j}(s) \frac{d s}{d \xi} d \xi
$$

The total electric and magnetic field, rspectively is obtained by contributing all wire segments [4]:

$$
\begin{gathered}
\vec{E}^{m}=\frac{1}{j 4 \pi \omega \varepsilon_{0}} \sum_{m=1}^{M} \sum_{i=1}^{n_{e}}\left[k^{2} \int_{-1}^{1} \vec{e}_{s^{\prime}} I_{i}^{m} f_{i}(\xi) g_{0}\left(\vec{r}, \vec{r}^{\prime}\right) \frac{d s^{\prime}}{d \xi} d \xi+\int_{-1}^{1} I_{i}^{m} \frac{\partial f_{i}(\xi)}{\partial \xi} \nabla g_{0}\left(\vec{r}, \vec{r}^{\prime}\right) \frac{d s^{\prime}}{d \xi} d \xi\right] \\
\vec{H}^{m}=-\frac{1}{4 \pi} \sum_{m=1}^{M} \sum_{i=1}^{n_{e}} \int_{-1}^{1} I_{i}^{m} f_{i}(\xi) \vec{e}_{s^{\prime}} \times \nabla g_{0}\left(\vec{r}, \vec{r}^{\prime}\right) \frac{d s^{\prime}}{d \xi} d \xi
\end{gathered}
$$

where $N$ and $n$ stands for the actual number of elements and local nodes, respectively.

\section{Computational examples}

The first example is related to the circular loop antenna insulated in free space and excited via unit voltage source at, $\varphi=0^{\circ}$, as depicted in Figure 2. The loop radius is $a=0.0027 \lambda$ and the wire radius is $b=0.0637 \lambda$ at $3 \mathrm{GHz}$. Figures 3 and 4 show the current distribution along the loop. All calculations are carried out by using linear elements. The results computed by BEM are compared to the results obtained by the Moment Method (MoM) available from [6]. 


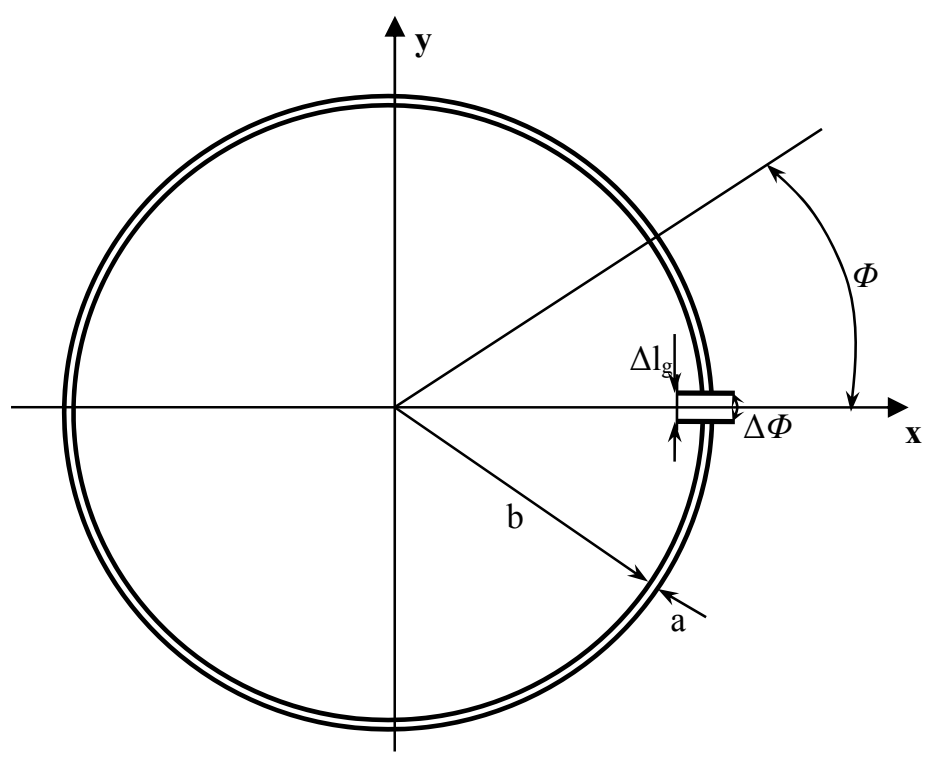

Figure 2: Thin wire loop antenna.

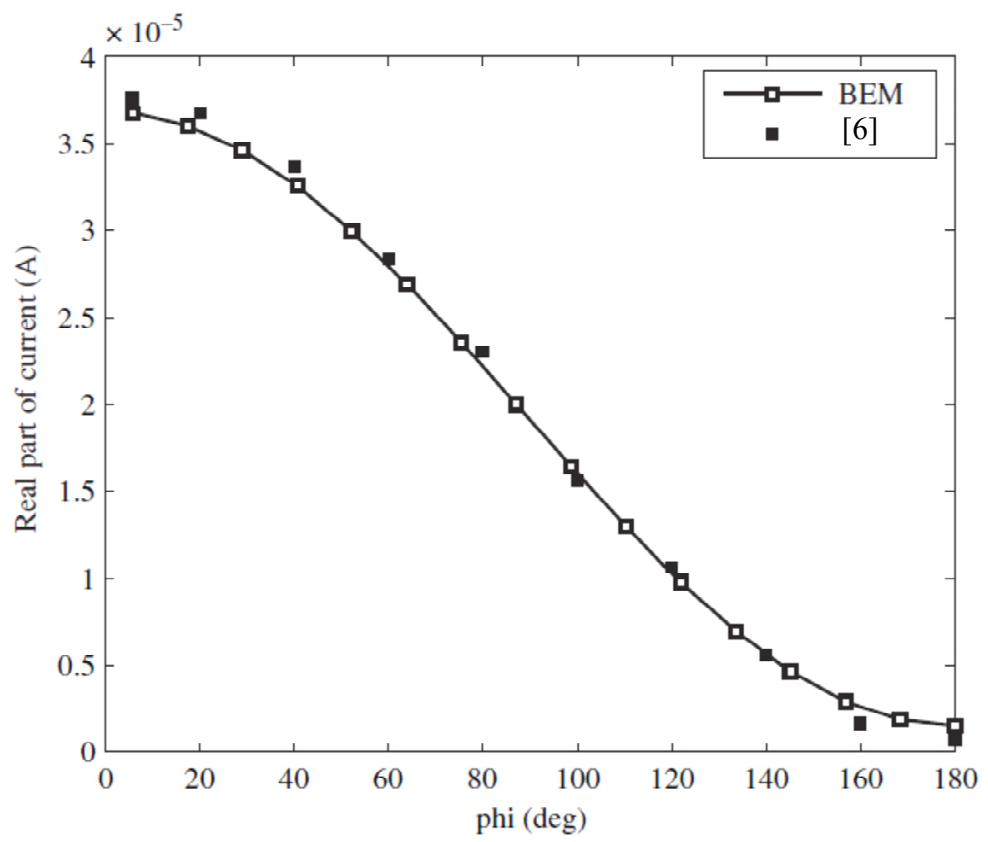

Figure 3: Real part of the loop current $(a=0.0027 \lambda, b=0.0637 \lambda, f=3 \mathrm{GHz})$. 


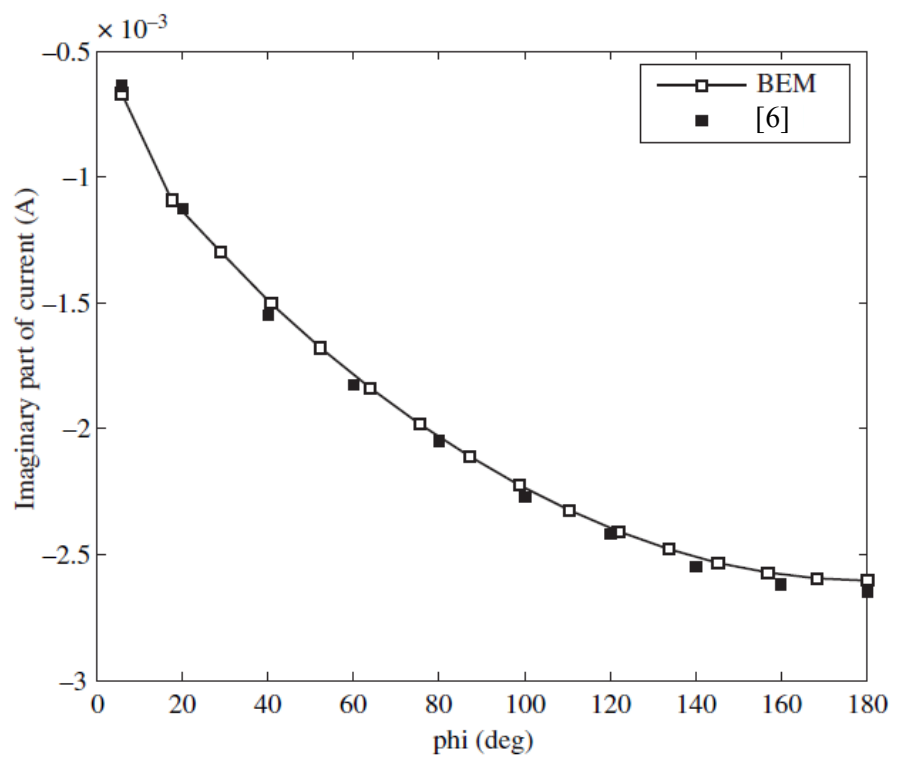

Figure 4: Imaginary part of loop current $(a=0.0027 \lambda, b=0.0637 \lambda, f=3 \mathrm{GHz})$.

The next set of computational examples is related to cylindrical helix depicted in Figure 5, while the amplitude of current distribution at frequency $f=30 \mathrm{MHz}$ and $750 \mathrm{MHz}$, respectively is shown in Figures 6 and 7. The cylindrical helix consists of 6 turns, radius $5 \mathrm{~cm}$, pitch angle $11^{\circ}$ and wire radius $1 \mathrm{~mm}$.

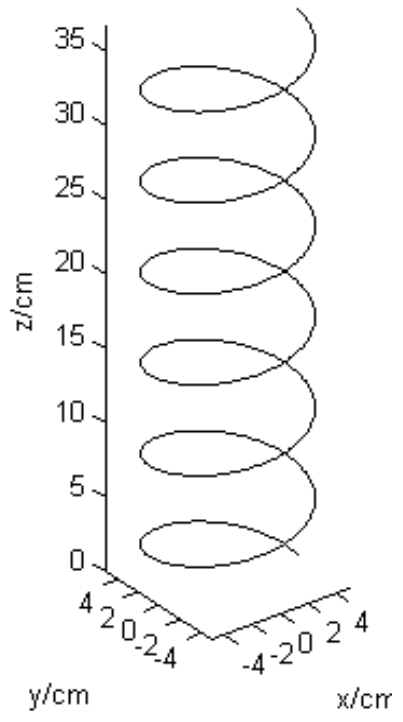

Figure 5: Geometry of cylindrical helix. 


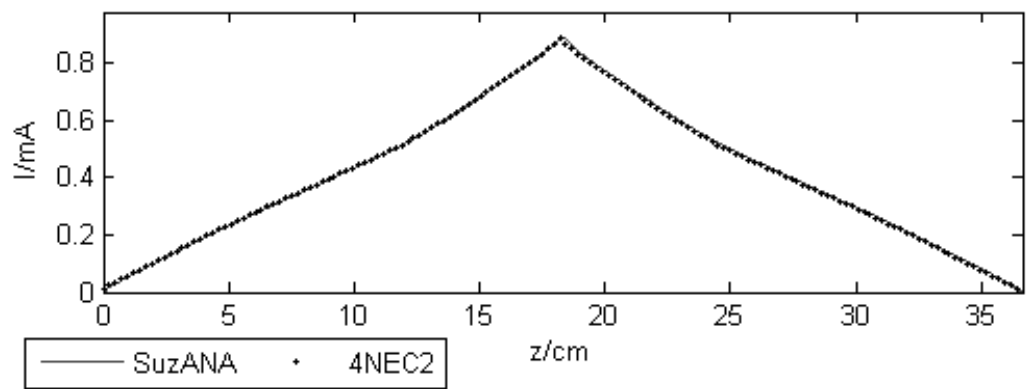

Figure 6: Amplitude of current distribution along cylindrical helix at $f=30 \mathrm{MHz}$.

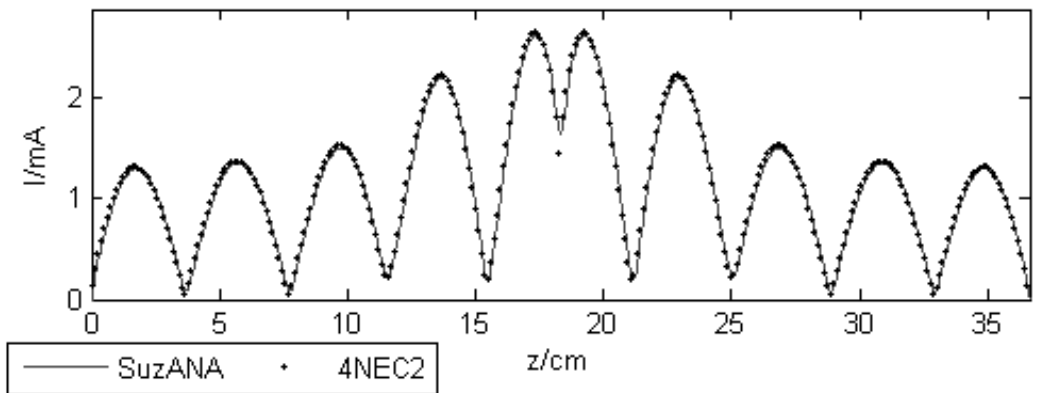

Figure 7: Amplitude of current distribution along cylindrical helix at $f=750 \mathrm{MHz}$.

The related radiation pattern of cylindrical helix in horizontal and vertical plane, respectively, at frequency $f=30 \mathrm{MHz}$ and $750 \mathrm{MHz}$ is shown in Figures 8 and 9.

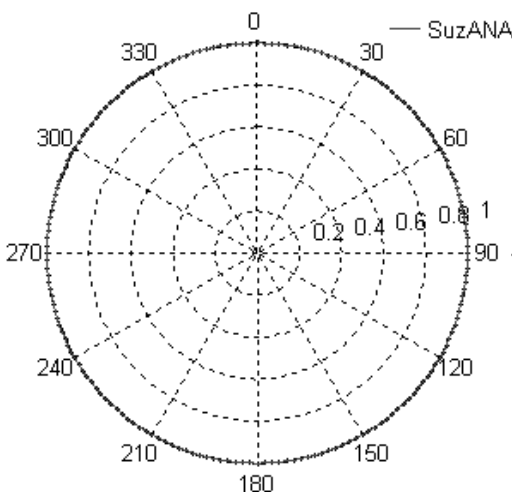

(a) Horizontal plane

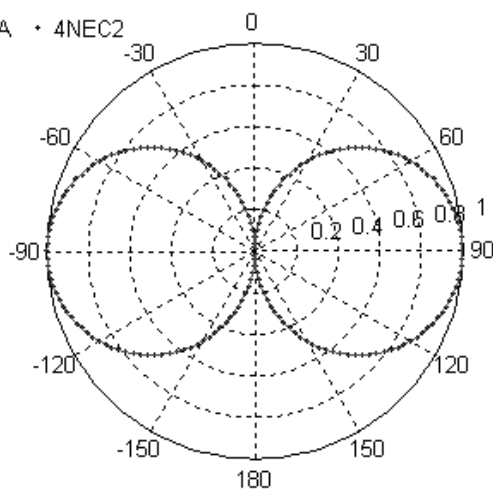

(b) Vertical plane

Figure 8: $\quad$ Radiation pattern of cylindrical helix at $f=30 \mathrm{MHz}$. 


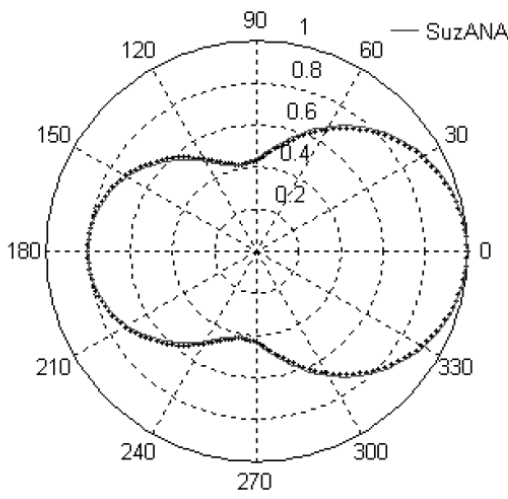

(a) Horizontal plane

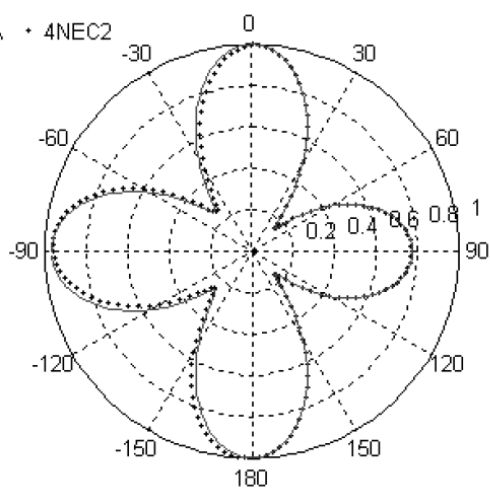

(b) Vertical plane

Figure 9: $\quad$ Radiation pattern of cylindrical helix at $f=750 \mathrm{MHz}$.

The numerical results for the current distribution obtained via GB-IBEM (computed via SuzANA code developed by the authors of this paper [7]) are in a satisfactory agreement with the results obtained via widely used NEC code [8].

Furthermore, the conical helix consisting of 9 turns, starting radius and pitch angle $5 \mathrm{~cm}$ and $12^{\circ}$, respectively, and wire radius $1 \mathrm{~mm}$, is analyzed. The amplitude of current distribution at frequency $f=1 \mathrm{GHz}$ is shown in Figure 11, while the corresponding radiation pattern is shown in Figure 12.

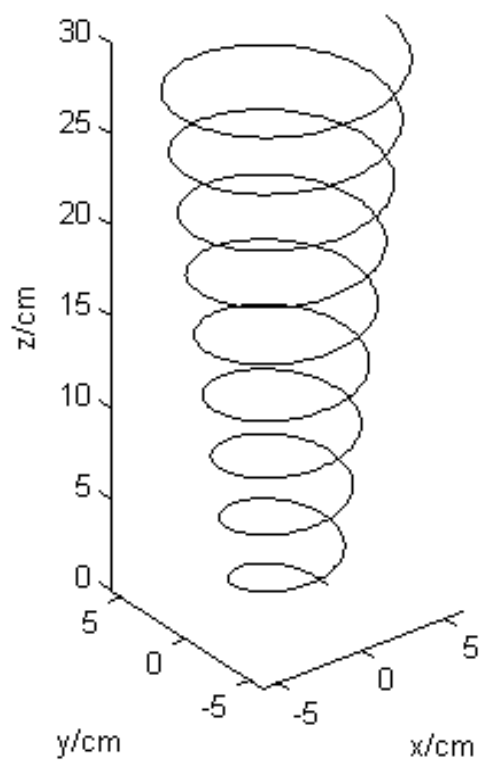

Figure 10: Geometry of conical helix. 


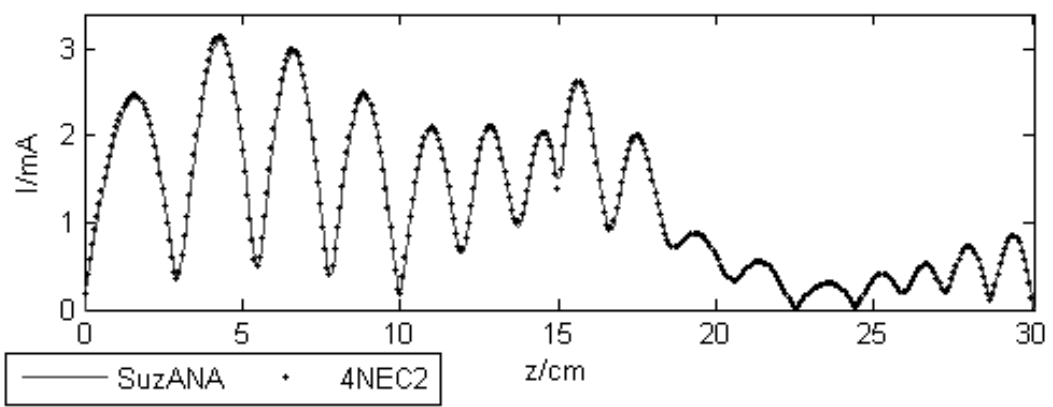

Figure 11: Amplitude of current distribution along conical helix at $f=1 \mathrm{GHz}$.

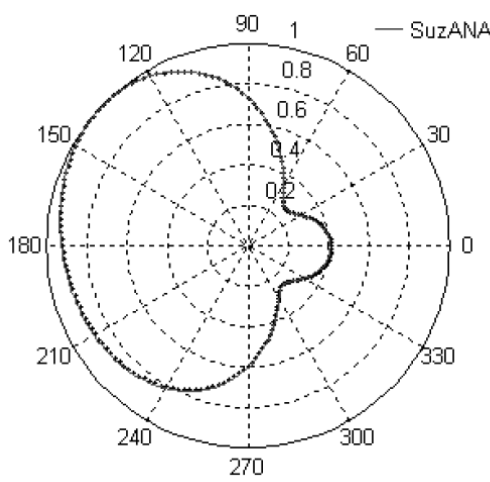

(a) Horizontal plane

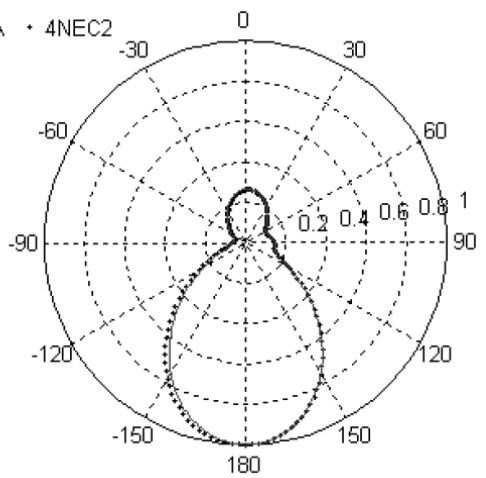

(b) Vertical plane

Figure 12: Radiation pattern of conical helix at $f=1 \mathrm{GHz}$.

Again, the results obtained via different approaches are in a rather satisfactory agreement. Next example is related to the spherical helix with helix radius $7.5 \mathrm{~cm}$ and pitch angle is $5^{\circ}$ and the wire radius $0.2 \mathrm{~mm}$, Figure 13 .

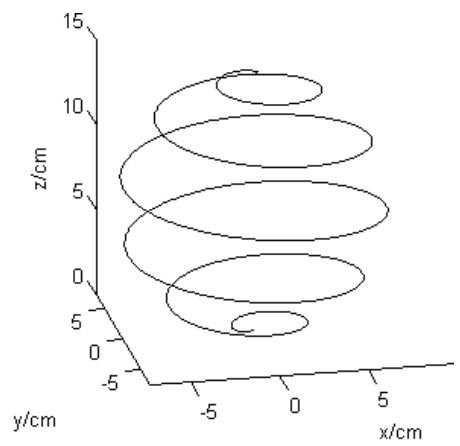

Figure 13: Geometry of spherical helix. 
The amplitude of current distribution at frequency $f=1 \mathrm{GHz}$ is shown in Figure 14, while the related radiation pattern is shown in Figure 15. The results obtained via different approaches are in a quite good agreement.

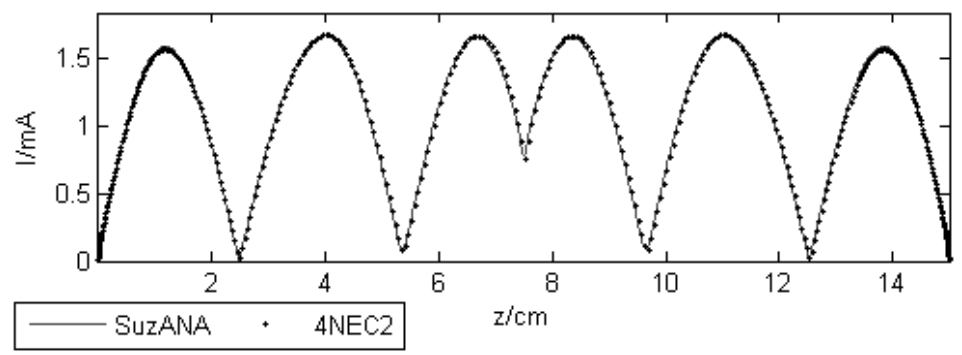

Figure 14: Amplitude of current distribution along the spherical helixat $f=500 \mathrm{MHz}$.

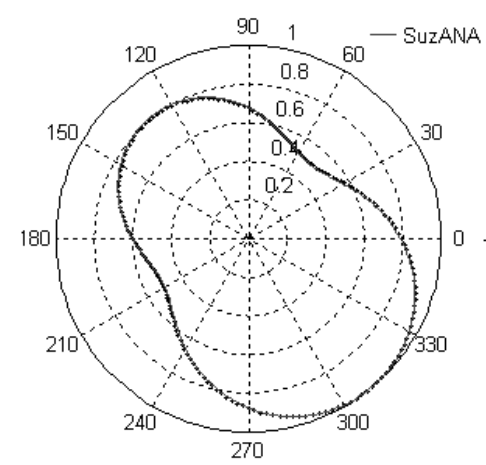

(a) Horizontal plane

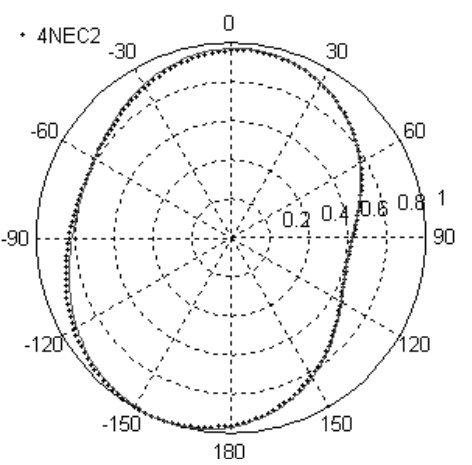

(b) Vertical plane

Figure 15: Radiation pattern of spherical helix at $f=500 \mathrm{MHz}$.

Finally, the last example deals with a multiple helix configuration presented in Figure 16. The helix at the center of coordinate system is the active antenna. The distance between the wire axes is $0.5 \mathrm{~m}$.

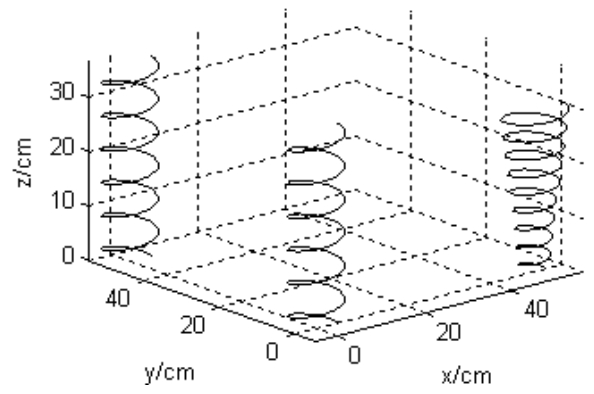

Figure 16: System of multiple helical antennas. 


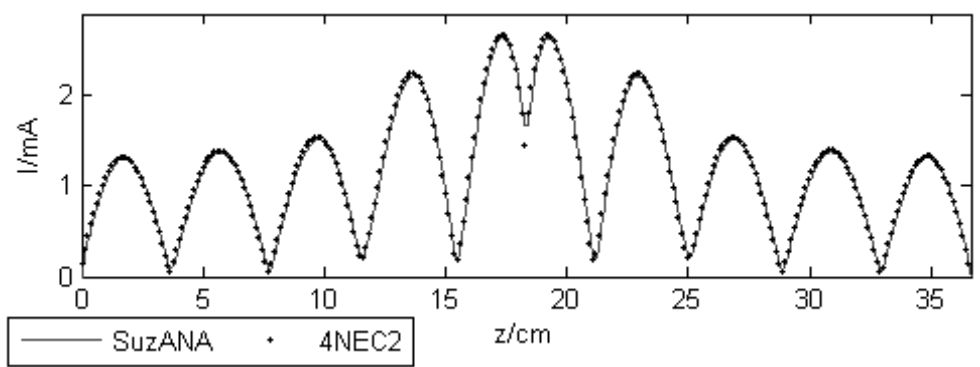

Figure 17: The current amplitude on active cylindrical helix at $f=750 \mathrm{MHz}$.

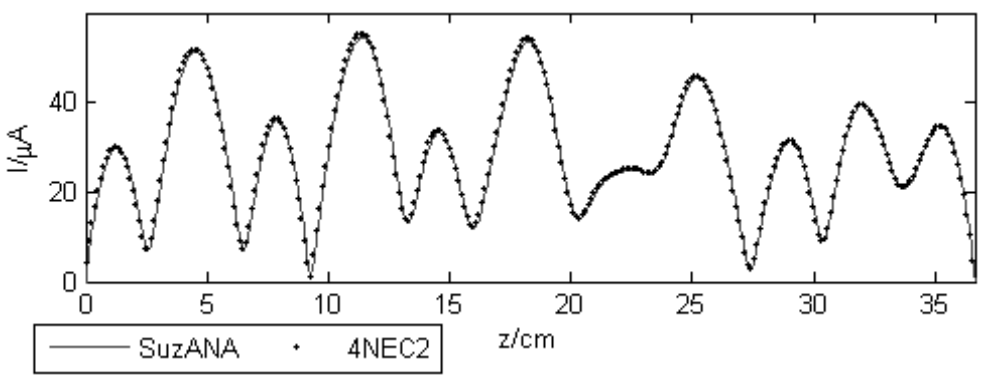

Figure 18: The current amplitude on passive cylindrical helix at $f=750 \mathrm{MHz}$.

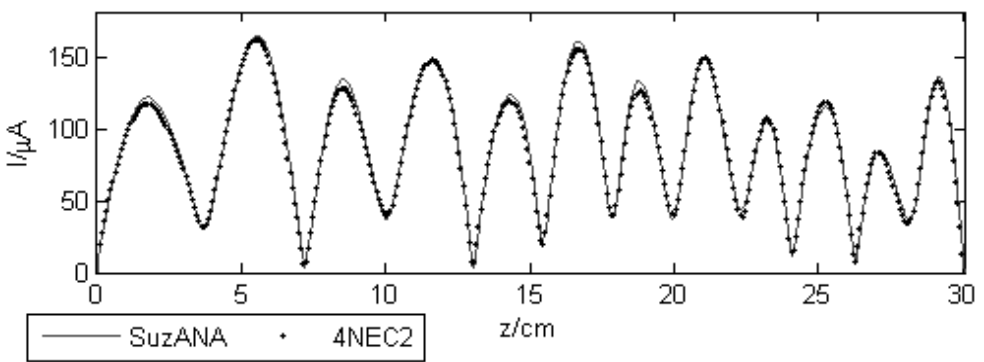

Figure 19: The current along passive conical helix at $f=750 \mathrm{MHz}$.

The results obtained via different techniques are still in a good agreement.

\section{Conclusion}

The analysis of curved wire antennas has been presented in this work. The formulation is based on the corresponding Pocklington integro-differential equation which is solved by the Galerkin-Bubnov scheme of the Indirect Boundary Element Method (GB-IBEM). Some illustrative computational examples pertaining to thin wire loop antenna and a few helix configurations are presented in the paper. The numerical results obtained by GB-IBEM agree satisfactorily with the results obtained NEC code. 


\section{References}

[1] W. L. Stutzman, Antenna theory and design, John Wiley \& Sons, Inc., 1981.

[2] C. A. Balanis, Antenna Theory: Analysis Design, Third Edition, John Wiley \& Sons, Inc., 2005.

[3] P. R. P. Hoole (Ed), Smart Antennas and Signal Processing for Communications, Biomedical and Radar Systems, WIT Press, SouthamptonBoston, 2001.

[4] D. Poljak, K. El Khamlichi Drissi, Electromagnetic Field Coupling to Overhead Wire Configurations: Antenna Model versus Transmission Line Approach, International Journal of Antennas and Propagation, pp. 1-18, 2012.

[5] D. Poljak, Advanced Modelling in Computational electromagnetic Compatibility, John Wiley and Sons, New York, 2007.

[6] Champagne II, N. J., Williams, J. T., Wilton, D. R., The Use of Curved Segments for Numerically Modeling Thin Wire Antennas and Scatterers, IEEE Trans. AP, Vol. 40, No. 6., pp. 682-688, June 1992.

[7] D. Poljak, V. Doric, S. Antonijevic, Computer Modeling of Wire Structures (in Croatian), Kigen, Zagreb, 2009.

[8] G. J. Burke et al. "Numerical electromagnetics code-a program for antenna system analysis," In: Proceedings of the International Symposium on Electromagnetic Compatibility, Rotterdam, The Netherlands, 1979. 